\title{
The relationship between socioeconomic indices and potentially zoonotic pathogens carried by wild Norway rats: a survey in Rhône, France (2010-2012)
}

\author{
F. AYRAL ${ }^{1 *}$, J. ARTOIS ${ }^{2}$, A.-L. ZILBER ${ }^{1}$, F. WIDÉN $^{3}$, K.C. POUNDER ${ }^{4}$, \\ D. AUBERT ${ }^{5}$, D. J. BICOUT ${ }^{6}$ AND M. ARTOIS ${ }^{1}$ \\ ${ }^{1}$ WildTech, PERS, Université de Lyon-VetAgro Sup, Marcy l'Etoile, France \\ ${ }^{2}$ Université de Rennes 2, Rennes Cedex, France \\ ${ }^{3}$ The National Veterinary Institute (SVA), Department of Virology, Immunobiology and Parasitology, Uppsala, \\ Sweden \\ ${ }^{4}$ Institute of Integrative Biology, University of Liverpool, Liverpool, UK \\ ${ }^{5}$ Laboratory of Parasitology, EA 3800, SFR CAP-Santé FED 4231, National Reference Centre on \\ Toxoplasmosis, University of Reims Champagne-Ardenne, Reims Cédex, France \\ ${ }^{6}$ Biomatématiques et Epidémiologie, EPSP-TIMC, UMR CNRS 5525, UFJ, VetAgro Sup, Marcy l'Etoile
}

Received 13 December 2013; Final revision 24 March 2014; Accepted 21 April 2014;

first published online 16 May 2014

\section{SUMMARY}

Leptospira interrogans, hantaviruses (particularly Seoul virus), hepatitis E virus (HEV), and Toxoplasma gondii are rat-associated zoonoses that are responsible for human morbidity and mortality worldwide. This study aimed to describe the infection patterns of these four pathogens in wild rats (Rattus norvegicus) across socioeconomic levels in neighbourhoods in Lyon, France. The infection or exposure status was determined using polymerase chain reaction or serology for 178 wild rats captured in 23 locations; additionally, confirmatory culture or mouse inoculation was performed. Multivariate logistic regression analyses were used to investigate whether morphological and socioeconomic data could predict the infection status of the rats. This study revealed that the rat colony's age structure may influence the prevalence of $L$. interrogans, hantavirus, and HEV. In addition, areas with high human population densities and low incomes may be associated with a greater number of infected rats and an increased risk of disease transmission.

Key words: Hepatitis E virus, Leptospira interrogans, Norway rats, Toxoplasma gondii, Seoul virus.

\section{INTRODUCTION}

Wildlife is estimated to be responsible for $72 \%$ of emerging infectious diseases in humans [1]. Key among wildlife species, Rattus norvegicus is prevalent worldwide and harbours rat-borne zoonoses (RBZs) [2], such as Leptospira interrogans, hantaviruses,

\footnotetext{
* Author for correspondence: Dr. F. Ayral, 1 avenue Bourgelat, 69280 Marcy l'Etoile, France.

(Email: florence.ayral@vetagro-sup.fr)
}

hepatitis E virus (HEV), and Toxoplasma gondii. These RBZs are responsible for potentially lifethreatening human infections and have raised public health concerns in Europe.

Leptospirosis (caused by L. interrogans sensu lato), an acute bacterial infection in humans, was recently recognized as an emerging public health problem [3]. Leptospira are maintained by a wide range of hosts, and $R$. norvegicus is reportedly the primary carrier [4]. The bacteria are shed in the hosts' urine and can survive for prolonged periods. Occupational 
and recreational exposures to contaminated water bodies in proximity to various host species are commonly reported risk factors. However, cities, particularly environments with poor hygiene, are the primary source of rat-associated leptospirosis [5].

Haemorrhagic fever with renal syndrome is a mild to severe human disease caused by viruses from the genus Hantavirus, family Bunyaviridae [6]. Each hantavirus species is carried by a specific rodent host species; Seoul virus (SEOV) has been found to be carried by $R$. norvegicus worldwide [2]. Unlike in Asia, few confirmed occurrences were reported in Europe before the recent emergence in the UK and France [7, 8]. Hantavirus is shed in the excreta of infected hosts, and the primary transmission pathways are the inhalation of suspensions of contaminated dust and the passage of infected saliva through bites [9]. Areas with growing rodent populations are at increased risk for human infection [10].

HEV is widespread and causes acute hepatitis in humans. A variety of HEV strains have been identified in Norway rats from Europe without any evidence of zoonotic pathogenicity [11, 12]. However, zoonotic HEV has been reported in the USA [13], confusing the role of rats as a disease reservoir for humans and livestock.

Toxoplasmosis is a highly prevalent parasitic disease worldwide and is caused by the protozoan T. gondii. Humans become infected after ingesting food contaminated with $T$. gondii oocysts. Vertical transmission is particularly concerning for humans, as in utero infection can cause abortion or congenital defects [14]. Although cats are the only hosts known to excrete the infectious form, the parasite cycle involves most warm-blooded vertebrate species, including Norway rats. As felids' prey, rats are important in the parasite life cycle and in human toxoplasmosis epidemiology.

The Norway rat is a synanthropic species that uses human habitats for shelter and food. Impoverished inner cities have been targeted for RBZ investigations because it is assumed that poor hygiene conditions, infrastructure disrepair, and low health status may lead to contact between rats and humans, and potentially to RBZ transmission [15]. Conditions in impoverished cities may also be related to high numbers of infected and shedder rats, which are responsible for rapidly spreading pathogens in human habitats and increased human exposure. However, no previous studies have investigated whether impoverished inner cities have a higher prevalence of infected rats.
A description of RBZ carriage in Norway rats across socioeconomic indices increases the knowledge of the related health risks. A more complete understanding of zoonotic infection in rats would mitigate their role in spreading zoonotic diseases by increasing awareness among healthcare professionals and supporting the development of appropriate surveillance.

The aims of the present study were (1) to describe the prevalence of the four pathogens in the Lyon region, France, (2) to identify the risk factors for infection in Norway rats, and (3) to define the potential socioeconomic indicators for human exposure in the Lyon region. Thus, trapping was performed on an urbanization gradient in and around Lyon. Multivariate models were built to predict the pathogen carriage status of the rats; morphological and socioeconomic data were used as independent variables.

\section{METHODS}

\section{Study area}

The study area comprised 23 locations in the Rhône department (i.e. administrative spatial unit) of France, which encompasses the city of Lyon and 14 farms within $30 \mathrm{~km}$ of our laboratory $\left(45^{\circ} 47^{\prime} 30.93^{\prime} \mathrm{N}\right.$; $4^{\circ} 42^{\prime} 30 \cdot 28^{\prime}$ E). This area contains a range of urbanization levels. Farms were requested to participate based on their inclusion in previous surveys, and all agreed. The dwellings and public areas were selected based on high rat concentrations reported by the Hygiene Service of Lyon.

Most farms were medium to large size (i.e. $>100$ animals) with mixed livestock. The nine urban trapping locations were distributed in dwelling areas, public gardens, waste disposal areas, and an industrial area in the city centre and suburbs.

\section{Sampling}

The survey was subdivided into 6-month periods: (1) September 2010 to February 2011 and (2) October 2011 to March 2012. To account for possible seasonal variation in the pathogen prevalence, the two periods encompassed the same season. The sampling size $(n \approx 90)$ was calculated using a $95 \%$ confidence level, a relative accuracy of $50 \%$, and an expected prevalence of $10-30 \%$ [16]. To account for possible variation in the pathogen prevalence among different urbanization levels, the same sample size was used 
in areas of low and high population density. During the two periods, 178 free-living Norway rats were trapped in areas of low $(n=94)$ and high $(n=84)$ population density.

Most rats were captured with small $(28 \mathrm{~cm} \times 9 \mathrm{~cm} \times$ $9 \mathrm{~cm})$ or large $(50 \mathrm{~cm} \times 15 \mathrm{~cm} \times 15 \mathrm{~cm})$ single-catch rat traps. The captured rats were transported to the laboratory and immediately anaesthetized using isoflurane. A blood sample was obtained by cardiac puncture. Subsequently, the rats were sacrificed by cervical dislocation. The rats that succumbed to capture were frozen at $-20^{\circ} \mathrm{C}$ and thawed on the day of dissection. Data were recorded on weight, size, sex, sexual maturity (i.e. the presence of seminal vesicles in males and a developed uterus in females) and pregnancy. Lung and liver lobe fragments weighing between 50 and $100 \mathrm{mg}$ were collected, and the kidney was removed. The heart was incorporated in an antibiotic solution with $1.2 \times 10^{-5} \mathrm{~g} / \mathrm{ml}$ amoxicillin (Amoxicilline Panpharma, France), $120 \mathrm{UI} / \mathrm{ml}$ penicillin and $120 \mu \mathrm{g} / \mathrm{ml}$ streptomycin (Pen-Strep Liq 10000 , Gibco, USA). Faecal samples were collected from the rectum, and all tissue and faecal samples were stored at $-80^{\circ} \mathrm{C}$ immediately following collection.

The authors assert that all procedures contributing to this work comply with the ethical standards of the relevant national and institutional guides on the care and use of laboratory animals (agreement no. 69-020931).

\section{Screening protocols}

L. interrogans colonization of the kidney was assessed via culture [17] and a pathogen-specific L. interrogans TaqMan real-time polymerase chain reaction (PCR) kit (TaqVet PathoLept kit, LSI, France); additionally, the $r р о B$ gene was targeted. Lung tissues were screened for hantavirus using a nested pan-hantavirus reverse transcription (RT)-PCR selective for partial polymerase large segment (L) gene sequences, subsequent molecular typing was performed to determine the hantavirus species as previously described [18]. The HEV viral load in the liver and faeces was analysed using TaqMan real-time PCR reagents specific to rat-strain HEV and genotypes 1-4 [19]. Sera extracted from blood-soaked blotters were tested for T. gondii antibodies using a modified agglutination test (MAT) that detects $T$. gondii-specific IgG antibodies [20]. The hearts of animals with a positive agglutination (starting dilution 1:6) were bioassayed in mice to isolate viable $T$. gondii [21].

\section{Prevalence calculation}

L. interrogans, SEOV, and HEV were screened based detecting pathogen nucleic acid sequences or the microorganism itself; rats testing positive were considered to be infected and potential shedders of the pathogens. The rats with MAT-positive results were considered to be exposed because the presence of antibodies against the pathogen could result from previous or current contact with the pathogen. The prevalence of infected or exposed rats was defined as the proportion of rats with any positive test results. The apparent prevalence was restricted to the population sampled since and the administrative unit of the Rhône department

\section{Variables}

Two categories of explanatory covariates were considered: rat-related and socioeconomic covariates. Rat survival during the trapping was also considered, as death and freeze-thaw cycles before dissection could alter the sample quality and underestimate the prevalence.

The rat data included the species (determined by external morphology and macroscopic observations), sex, weight, size, and approximate age, based on sexual maturity and pregnancy. A body mass metric was constructed from the residuals that resulted from the regression of weight on size [22]. The capture success, a proxy of the population size, was accurately defined in five sites by

capture success $=\frac{n_{i}}{t_{i} \times d_{i}}$,

where $n_{i}$ is the number of rats captured, $t_{i}$ the number of traps set and $d_{i}$ the number of days of trapping at the site $i$.

The extent and features of urbanization were characterized using the socioeconomic data. Eleven candidate variables were extracted from the 2009 national census data provided by the Institut National de la Statistique et des Etudes Economiques. Remotely sensed data were obtained at the smallest geographical unit, which is the Ilot Regroupé pour l'Information Statistique (IRIS). Each IRIS contains 2000 inhabitants. The socioeconomic variables are displayed in Table 1.

\section{Spatial analysis}

The location and number of rats in each trapping area that tested positive and negative were mapped using 
Table 1. Description of socioeconomic covariates

\begin{tabular}{|c|c|c|}
\hline Covariates & Name of covariate & Definition \\
\hline Human population density & Human population & Population/surface $(\mathrm{km})^{2}$ \\
\hline Median income of the population & Income & Median incomes of the population included in the IRIS \\
\hline $\begin{array}{l}\text { Population growth from } 1999 \text { to } \\
2009\end{array}$ & Population growth & Population in 2009/population in 1999 \\
\hline $\begin{array}{l}\text { Percentage of } 20-64 \text { years age } \\
\text { group }\end{array}$ & Population age & 20-64 years age group/total population \\
\hline Percentage of flats & Per cent flat & Number of flats/number of flats + houses \\
\hline Percentage of small flats & Per cent small flat & Number of 1- and 2-room dwellings/number of dwellings \\
\hline Ratio of renters and owners & Ratio renter/owner & Number of renters/number of owner of their residency \\
\hline $\begin{array}{l}\text { Ratio of low-income dwellings } \\
\text { (LID) }\end{array}$ & ratio LID & Number of LID rented/number of other rented dwellings \\
\hline $\begin{array}{l}\text { Percentage of ungraduated } \\
\text { population }\end{array}$ & Level of graduation & $\begin{array}{l}\text { Population aged }>15 \text { years without a diploma/population aged } \\
>15 \text { years }\end{array}$ \\
\hline Ratio of large and small families & Family size & $\begin{array}{l}\text { Family with at least three children/family with two or fewer } \\
\text { children }\end{array}$ \\
\hline Ratio of unemployment & $\begin{array}{l}\text { Rate of } \\
\text { unemployment }\end{array}$ & Number of unemployed people/number of employed people \\
\hline
\end{tabular}

ArcGIS version 9.3 (ESRI, USA). Spatial clusters of high and low pathogen prevalence were identified using the Getis-Ord Gi* statistic. The local sum for features and the feature's neighbours were compared proportionally to the sum of all features. When the local sum was statistically significantly different from the expectation, the region was denoted as having low or high prevalence.

To identify the potential socioeconomic proxies of RBZ, the candidate variables from the census data sets were extracted from the IRIS units with trapping locations. An approach using various boundaries for the quadrats when extracting data was implemented to verify the effects of different spatial scales on the risk factors [23]. The socioeconomic data were extracted using four quadrat sizes: $100 \mathrm{~m} \times 100 \mathrm{~m}$, $200 \mathrm{~m} \times 200 \mathrm{~m}, \quad 400 \mathrm{~m} \times 400 \mathrm{~m}$, and $800 \mathrm{~m} \times 800 \mathrm{~m}$. The socioeconomic metrics for each quadrat were calculated by weighting the remotely sensed data by the percentage of the IRIS surface that was included in the quadrat. For each spatial level of analysis, a new data set was obtained by spatial query using the software $\mathrm{R}$ project version 3.0.1 (R Development Core Team, Austria) and ArcGIS version 9.3 (ESRI, Redland, CA, USA).

\section{Statistical analysis}

To identify the characteristics associated with each pathogen's carriage status, distributions of the explanatory variables were examined within the samples testing positive and negative. $\chi^{2}$ tests and Wilcoxon tests were used, as appropriate, and an alpha level of 0.05 was used to reject the null hypothesis. For each pathogen, the potential effect of the population size on the number of infected rats in the trapping sites was assessed by testing the Pearson correlation between the capture success and the related number of infected rats. An $\alpha$-level of 0.05 was used to reject the absence of correlation. The result obtained determined whether the capture success had to be considered in the models.

Statistical modelling was performed to evaluate the use of rat-related criteria and socioeconomic indices as predictors of exposure or infection in rats. All remotely sensed data were transformed to be normally distributed when they were incorporated into the models, and missing data were not considered. Simple logistic regression (SLR) was used to examine the relationship between each pathogen's prevalence and the explanatory variables. Covariates that were significantly associated with pathogen infection or exposure at an $\alpha$-level of $0 \cdot 10$ were considered for further analysis. To select independent variables, rat-related covariates were tested for multicollinearity using Spearman's rank correlation $(\rho>0 \cdot 7)$ with the Hmisc package in $\mathrm{R}$, whereas socioeconomic covariates were described with a principal component analysis (PCA) using the FactoMineR package in R. From the PCA result, one variable per main axis was chosen based on the comprehensiveness of the data. Selected covariates were incorporated into multivariate models. 


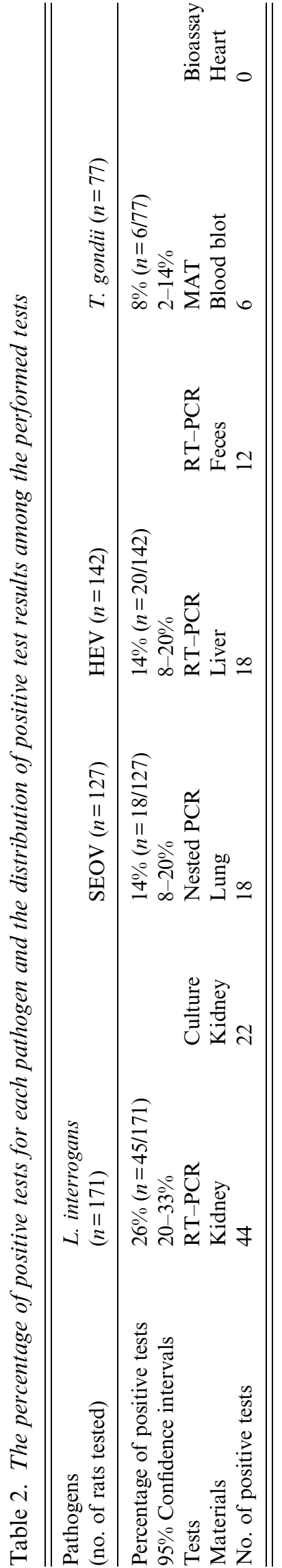

The variation inflation factors were calculated with the car package in $\mathrm{R}$ to verify a low effect of potential collinearity $(<5)$.

When the effect of rat death during capture was significantly associated with the infection status in SLRs, the data set was adjusted to account for a possible sample alteration effect.

For each pathogen, two multivariate logistic regression models were implemented. The selected covariates were incorporated into a generalized linear model (GLM) as fixed effects. The final GLM was selected using Aikake's Information Criterion (AIC) to balance model fit and parsimony. Then, the adjusted effects of the variables included in the final GLM were estimated with a generalized linear mixed model (GLMM) to control for the random effect of trapping locations and to account for the potential effects of clustering.

The remotely sensed data extracted from the quadrats were considered for each socioeconomic variable included in the final GLMs. A new set of models was created to account for the possible influence of changing spatial scales on the adjusted effects.

\section{RESULTS}

\section{Rat population}

During the two collection periods (37 and 39 days), 178 rats were trapped and sampled (54\% male). About two-thirds (63\%) of the rats were adults, and 18 of the 58 adult females were pregnant $(31 \%)$. The ratio of sexually immature to mature rats was similar between the farm $($ ratio $=40: 54)$ and city centre (ratio $=43: 58)$ sites. However, at the city centre sites, the ratio was lower in dwellings (ratio $=5: 37$ ) than in industrial areas (ratio $=19: 4)$.

\section{Pathogen detection}

Norway rats were the primary carrier and potential shedder of $L$. interrogans ( $26 \%$ prevalence), SEOV $(14 \%)$, and rat-specific-HEV (14\%). Eight per cent of the rats with a MAT titre of $\geqslant 1: 6$ were previously exposed to T. gondii. Among the 77 hearts, 58 were bioassayed in mice. Twenty-four bioassays were inconclusive because the mice did not survive past $48 \mathrm{~h}$ post-infection, and $T$. gondii was not isolated from the 34 other bioassays (Table 2).

Among 120 Norway rats screened for $L$. interrogans, SEOV, and HEV, 50 (42\%) were infected with at least one pathogen. A single pathogen was detected 
in the majority of infected rats $(n=31 / 50)$; the rest were co-infected with at least two agents. The proportion of rats infected with SEOV or HEV $(n=34)$ and co-infected with $L$. interrogans $(n=19)$ was significantly higher than the proportion of rats infected by L. interrogans alone (Pearson's $\chi^{2}=14 \cdot 63$, D.F. $=1$, $P=0.0001$ ); the odds ratio (OR) of being co-infected by $L$. interrogans and SEOV or HEV was 5.54 [95\% confidence interval (CI) 2.1-14.4]. Three of the infected rats were simultaneously carrying $L$. interrogans, SEOV, and HEV (see Supplementary online material).

The percentage of infected rats at the trapping sites varied: $0-65 \%$ for $L$. interrogans, $0-30 \%$ for SEOV, $0-61 \%$ for HEV, and $0-25 \%$ for $T$. gondii. The spatial distribution of sites with significantly higher or lower infected rats differed among the four pathogens. Nevertheless, high prevalence clusters were encountered for each pathogen in the Lyon region (Fig. 1).

For each pathogen, Pearson's coefficient which characterized the relationship between the capture success and the number of infected rats was not significantly different from zero (all $P$ values $>0 \cdot 14$ ). Thus, no correlation between the capture success and the number of infected individuals could be demonstrated.

\section{Characterization of infection correlates in rats}

\section{L. interrogans carriage}

From the SLR models, the OR of testing positive for $L$. interrogans was greater in sexually mature rats than in sexually immature rats. However, no significant relationship was observed between the infected rats and gender, body condition, or pregnancy (Table 3). The relationship between 10 normalized socioeconomic covariates and $L$. interrogans positivity was statistically significant (all $P$ values $\leqslant 0 \cdot 05$ ).

The multivariate analyses incorporated sexual maturity, human population density (HPD), and median income as independent covariates (multicollinearity test results are not shown). In the final GLM, the OR of being $L$. interrogans-positive was greater in adults than juveniles (OR 4.0, 95\% CI 1·6-9.9), and the OR increased with increasing normalized HPD (OR 1.8, 95\% CI 1.2-2.7) and decreasing normalized income (OR 0.6, 95\% CI 0.4-0.9). The relationship between these variables and $L$. interrogans positivity aligned but with decreased magnitude compared to the SLR. After controlling for clustering trapping locations using GLMMs, the ORs were nearly identical to the ORs obtained with a GLM. The estimated variance for the random effect of trapping locations was $0.007 \%$ of the total residual variance. The GLM was maintained for further analysis and used as the simplest model.

Among 155 rats analysed, 30\% $(n=47)$ died after trapping and were frozen and thawed before dissection. The OR of being $L$. interrogans-positive was greater for the surviving rats and the fresh samples compared to the frozen rats. Because there was a strong correlation between rat freezing and rat survival in the field, the effects of freezing and survival could not be differentiated. After adjusting the model for rat survival, the relationships between explanatory variables and $L$. interrogans positivity were similar to the results from the model that was not adjusted for survival. The relationships from the adjusted model were as follows: sexual maturity (OR $3 \cdot 4$, 95\% CI 1.3-8.5), normalized HPD (OR 1.9, $95 \%$ CI $1 \cdot 2-2 \cdot 8$ ), and normalized income (OR $0 \cdot 6$, $95 \%$ CI $0 \cdot 4-0 \cdot 8)$.

\section{SEOV carriage}

The results from the SLR models suggested that the OR of being SEOV-positive was greater in sexually mature rats than in sexually immature rats. There was no significant difference in the relationships between body condition, gender or pregnancy, and SEOV positivity. The relationships between seven normalized socioeconomic covariates and SEOV positivity were statistically significant (all $P$ values $\leqslant 0 \cdot 05$ ).

The multivariate analyses incorporated sexual maturity, HPD, and income as independent covariates. In the final GLM, sexual maturity and the normalized incomes of the population were retained. The OR of being SEOV-positive increased with decreasing normalized income (OR $0 \cdot 5,95 \%$ CI $0 \cdot 3-0 \cdot 8$ ) and appeared to be greater in adults than juveniles, although not statistically significant (OR 6.2, 95\% CI 1.1-34) for the latter group. In bivariate analyses, the relationship between these variables and SEOV positivity aligned but with increased magnitude. Similar results were obtained by accounting for clustering of the trapping locations in a GLMM. In the selected GLMM, the estimated variance for the random effect of trapping locations was $8 \cdot 88$, accounting for $99 \%$ of the total residual variance.

\section{HEV carriage}

The results from the SLR models suggested that the OR of being HEV-positive was greater in sexually 

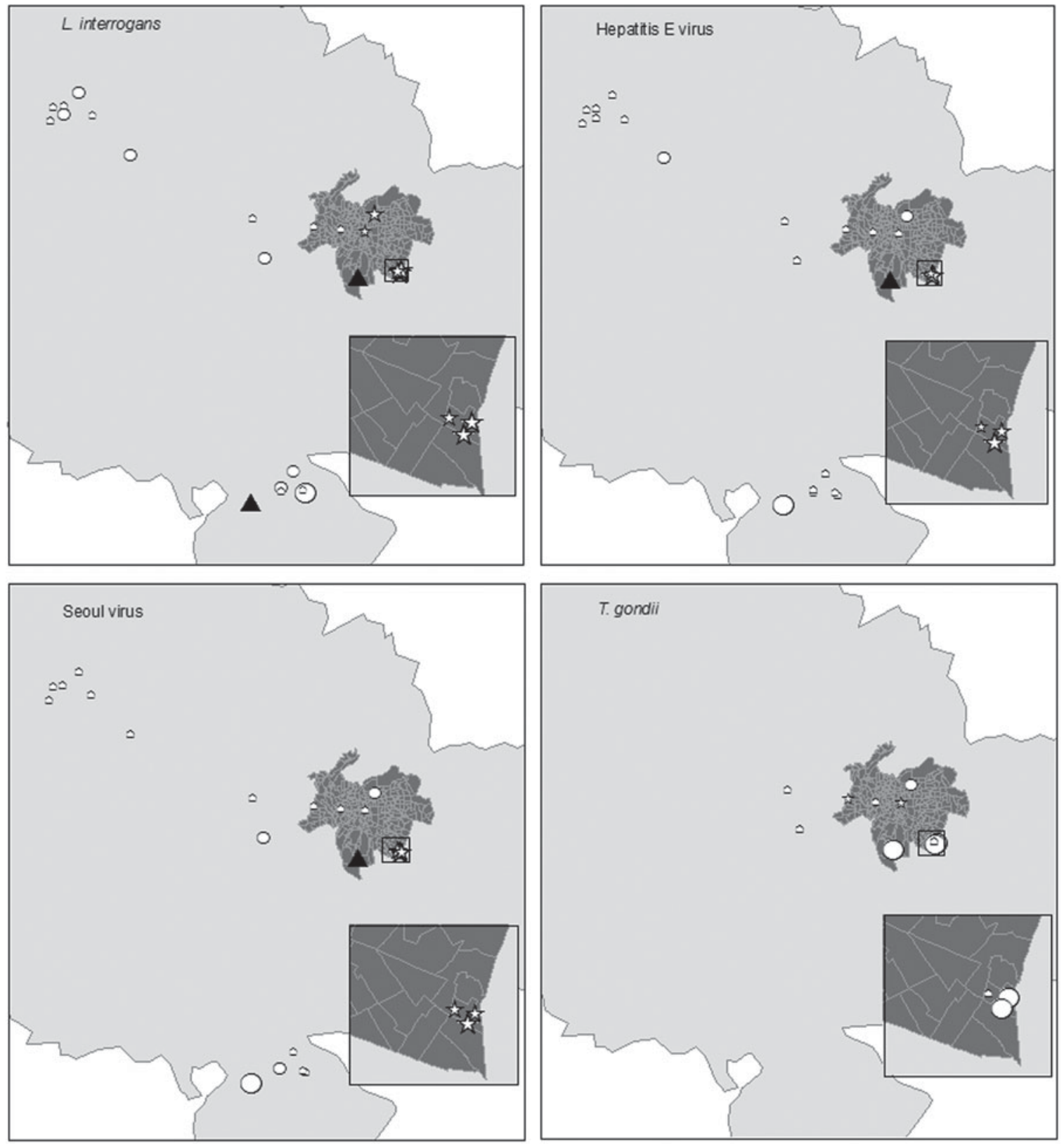

Distribution of infected rats in the Rhône including the city of Lyon

\section{$\frac{\text { Sample size } e^{*}}{\text { Small Medium Large }}$}

th th th High level of infected rats

- $\Delta \quad \Delta$ Low level of infected rats

- $\bigcirc$ Not significant

Lyon city centre

Rhône department

*Sample size: small=[1-6] rats; middle $=[7-12]$ rats; large $=[13-23]$ rats

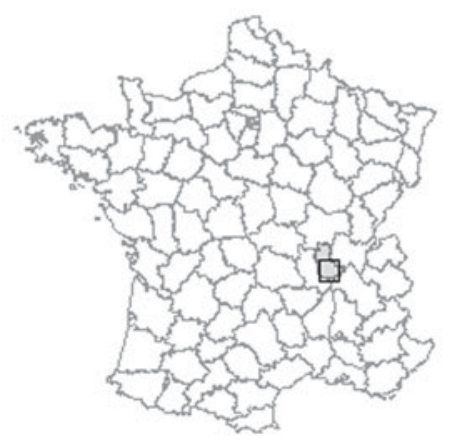

Fig. 1. The distribution of clusters of low (full triangle) and high (star) prevalences of the four pathogens detected in rats trapped in the Rhône department, France. 
Table 3. Unadjusted and adjusted odds ratios for testing positive for Leptospira interrogans, SEOV, HEV or Toxoplasma gondii among Norway rats

\begin{tabular}{|c|c|c|c|c|c|c|c|c|c|c|c|c|c|}
\hline \multirow[b]{2}{*}{ Covariates } & \multirow[b]{2}{*}{ Modes } & \multicolumn{3}{|c|}{ L. interrogans status $(n=171)$} & \multicolumn{3}{|c|}{ SEOV status $(n=127)$} & \multicolumn{3}{|c|}{ HEV status $(n=142)$} & \multicolumn{3}{|c|}{$T$ gondii status $(n=77)$} \\
\hline & & $\begin{array}{l}\text { SLR } \\
\text { OR }\end{array}$ & $\begin{array}{l}\text { GLM } \\
\text { aOR }\end{array}$ & $\begin{array}{l}\text { GLMM* } \\
\text { aOR }\end{array}$ & $\begin{array}{l}\text { SLR } \\
\text { OR }\end{array}$ & $\begin{array}{l}\text { GLM } \\
\text { aOR }\end{array}$ & $\begin{array}{l}\text { GLMM } \dagger \\
\operatorname{aOR}\end{array}$ & $\begin{array}{l}\text { SLR } \\
\text { OR }\end{array}$ & $\begin{array}{l}\text { GLM } \\
\text { aOR }\end{array}$ & $\begin{array}{l}\text { GLMM } \\
\text { aOR }\end{array}$ & $\begin{array}{l}\text { SLR } \\
\text { OR }\end{array}$ & $\begin{array}{l}\text { GLM } \\
\text { aOR }\end{array}$ & $\begin{array}{l}\text { GLMM } \\
\text { aOR }\end{array}$ \\
\hline \multirow[t]{2}{*}{ Survival after trapping } & Dead & Ref. & & & Ref. & & & Ref. & & & n.a. & & \\
\hline & Alive & $2(1 \cdot 1-3 \cdot 6)$ & - & - & $2 \cdot 2(0 \cdot 9-5 \cdot 2)$ & - & - & $1 \cdot 2(0.5-2 \cdot 8)$ & - & - & - & - & - \\
\hline \multirow{2}{*}{ Rat preservatives } & Frozen & Ref. & & & Ref & & & Ref. & & & n.a. & & \\
\hline & Fresh & $3.3(1.5-7 \cdot 4)$ & - & - & $1 \cdot 7(0 \cdot 6-4 \cdot 5)$ & - & - & $1 \cdot 2(0 \cdot 5-3 \cdot 1)$ & - & - & - & - & - \\
\hline \multirow[t]{2}{*}{ Sex } & Female & Ref. & & & Ref & & & Ref. & & & n.a. & & \\
\hline & Male & $1 \cdot 7(0 \cdot 9-3 \cdot 2)$ & - & - & $1.5(0.6-3.6)$ & - & - & $1(0 \cdot 4-2 \cdot 4)$ & - & - & - & - & - \\
\hline \multirow[t]{2}{*}{ Maturity } & Juvenile & Ref. & Ref. & & Ref. & & & Ref. & & & n.a. & & \\
\hline & Adult & $7 \cdot 3(3 \cdot 1-16 \cdot 6)$ & $4 \cdot 0(1 \cdot 6-9 \cdot 9)$ & $4 \cdot 0(1 \cdot 3-11 \cdot 9)$ & $9(1 \cdot 6-55)$ & $6 \cdot 2(1 \cdot 1-34)$ & $10 \cdot 5(0 \cdot 8-138)$ & $4 \cdot 4(1 \cdot 5-14 \cdot 3)$ & $2 \cdot 2(0 \cdot 7-7)$ & $2 \cdot 6(0 \cdot 5-14 \cdot 7)$ & - & - & - \\
\hline \multirow[t]{2}{*}{ Pregnancy } & No & Ref. & & & Ref. & & & Ref. & & & n.a. & & \\
\hline & Yes & $1 \cdot 5(0 \cdot 5-4 \cdot 1)$ & & & $0 \cdot 4(0 \cdot 1-2 \cdot 5)$ & - & - & $1 \cdot 1(0 \cdot 2-5)$ & - & - & - & - & - \\
\hline Body condition & & $1(0 \cdot 9-1 \cdot 0)$ & - & - & $1(0 \cdot 9-1)$ & - & - & $0 \cdot 9(0 \cdot 9-1 \cdot 0)$ & - & - & $1.02(1.01-1.03)$ & - & - \\
\hline Human population & & $3 \cdot 2(2 \cdot 3-4 \cdot 3)$ & $1.8(1 \cdot 2-2 \cdot 7)$ & $1.8(1.1-2.9)$ & $1.9(1 \cdot 3-2 \cdot 7)$ & - & - & $1 \cdot 7(1 \cdot 2-2 \cdot 4)$ & - & - & $1 \cdot 2(0 \cdot 7-2 \cdot 3)$ & - & - \\
\hline Incomes (median) & & $0 \cdot 3(0 \cdot 2-0 \cdot 4)$ & $0.6(0 \cdot 4-0.9)$ & $0 \cdot 6(0 \cdot 3-0 \cdot 9)$ & $0.4(0 \cdot 3-0.7)$ & $0.5(0 \cdot 3-0 \cdot 8)$ & $0 \cdot 4(0 \cdot 1-2 \cdot 1)$ & $0 \cdot 3(0 \cdot 2-0 \cdot 5)$ & $0 \cdot 4(0 \cdot 2-0 \cdot 6)$ & $0 \cdot 3(0 \cdot 1-0 \cdot 8)$ & $1 \cdot 3(0 \cdot 8-2 \cdot 2)$ & - & - \\
\hline Population expansion & & $1 \cdot 1(0 \cdot 8-1 \cdot 5)$ & - & - & $1 \cdot 4(0 \cdot 9-2 \cdot 3)$ & - & - & $1 \cdot 4(0 \cdot 9-2 \cdot 2)$ & - & - & $1.7(0.8-3.9)$ & - & - \\
\hline Population age & & $0.6(0.4-0.9)$ & - & - & $0 \cdot 2(0.02-1 \cdot 3)$ & - & - & $0 \cdot 1(0 \cdot 01-1 \cdot 2)$ & - & - & $0.7(0.4-1 \cdot 4)$ & - & - \\
\hline Per cent flat & & $2 \cdot 6(1.9-3 \cdot 6)$ & - & - & $1.6(0 \cdot 9-2.5)$ & - & - & $1.5(0.9-2.2)$ & - & - & $0.7(0.3-1 \cdot 6)$ & - & - \\
\hline Per cent small flat & & $3 \cdot 0(2 \cdot 1-4 \cdot 2)$ & - & - & $1.6(1 \cdot 1-2 \cdot 6)$ & - & - & $1.5(1.0-2 \cdot 3)$ & - & - & $1 \cdot 1(0 \cdot 4-2 \cdot 8)$ & - & - \\
\hline Ratio renter/owner & & $4 \cdot 2(2 \cdot 2-8 \cdot 0)$ & - & - & $0 \cdot 6(0 \cdot 3-1 \cdot 1)$ & - & - & $0.5(0 \cdot 3-1 \cdot 1)$ & - & - & $1.2(0.8-2 \cdot 0)$ & - & - \\
\hline $\begin{array}{l}\text { Ratio LID } \\
\text { late }\end{array}$ & & $3 \cdot 1(2 \cdot 3-4 \cdot 3)$ & - & - & $2 \cdot 7(\mathbf{1} \cdot 7-4 \cdot 2)$ & - & - & $2 \cdot 4(1.6-3 \cdot 6)$ & - & - & $0.9(0.5-1.6)$ & - & - \\
\hline Level of graduation & & $2 \cdot 8(2 \cdot 1-3 \cdot 8)$ & - & - & $2 \cdot 2(1 \cdot 5-3 \cdot 2)$ & - & - & $2 \cdot 8(1 \cdot 8-4 \cdot 1)$ & - & - & $0 \cdot 1(0 \cdot 0-7 \cdot 3)$ & - & - \\
\hline Family size & & $3 \cdot 1(2 \cdot 3-3 \cdot 4)$ & - & - & $2 \cdot 6(1 \cdot 7-4 \cdot 1)$ & - & - & $2.6(1.7-3.9)$ & - & - & $0.8(0.5-1.5)$ & - & - \\
\hline Rate of unemployment & & $3 \cdot 1(2 \cdot 3-4 \cdot 2)$ & - & - & $2 \cdot 3(1 \cdot 5-3 \cdot 4)$ & - & - & $2.5(1.7-3.8)$ & - & - & $0.8(0.5-1 \cdot 4)$ & - & - \\
\hline
\end{tabular}

SLR, Simple logistic regression; GLM, generalized linear model; GLMM, generalized linear mixed model; OR, odds ratio; aOR, adjusted odds ratio; n.a., not applicable; LID, low-income dwelling.

$*$ Variance of the random effect $=0 \cdot 007$.

$\dagger$ Variance of the random effect $=1 \cdot 7$.

$\$$ Variance of the random effect $=8 \cdot 9$.

All results in bold were significantly different from 1 at $\alpha \leqslant 0 \cdot 05$. 
Table 4. Adjusted odds ratios using the final GLM or GLMM and incorporating the human population density (HPD) and median income (MI) by quadrat $(a, b, c, d)$

\begin{tabular}{|c|c|c|c|c|c|c|c|c|c|c|}
\hline \multirow[b]{2}{*}{ Covariates } & & \multicolumn{3}{|c|}{ L. interrogans status* } & \multicolumn{3}{|c|}{ SEOV status $\dagger$} & \multicolumn{3}{|c|}{ HEV status: } \\
\hline & & $\mathrm{aOR}$ & $95 \% \mathrm{CI}$ & $P$ & $\mathrm{aOR}$ & $95 \% \mathrm{CI}$ & $P$ & $\mathrm{aOR}$ & $95 \% \mathrm{CI}$ & $P$ \\
\hline \multirow[t]{2}{*}{ Maturity } & Juveniles & Ref. & & & Ref. & & & Ref. & & \\
\hline & Adults & $3 \cdot 4$ & $(1 \cdot 4-8 \cdot 5)$ & 0.01 & $9 \cdot 3$ & $(0 \cdot 7-124)$ & $0 \cdot 09$ & $2 \cdot 1$ & $(0 \cdot 4-11 \cdot 4)$ & $0 \cdot 38$ \\
\hline HPD (a) & & $2 \cdot 0$ & $(1 \cdot 4-2 \cdot 9)$ & $0 \cdot 01$ & - & - & - & - & - & - \\
\hline MI (a) & & $0 \cdot 7$ & $(0 \cdot 5-1 \cdot 0)$ & $0 \cdot 19$ & $0 \cdot 4$ & $(0 \cdot 03-5 \cdot 8)$ & $0 \cdot 44$ & $0 \cdot 3$ & $(0 \cdot 1-0 \cdot 7)$ & $0 \cdot 007$ \\
\hline \multirow[t]{2}{*}{ Maturity } & Juveniles & Ref. & & & Ref. & & & Ref. & & \\
\hline & Adults & $3 \cdot 6$ & $(1 \cdot 8-8 \cdot 9)$ & 0.02 & $7 \cdot 9$ & $(0 \cdot 6-100)$ & $0 \cdot 11$ & $3 \cdot 9$ & $(0 \cdot 6-24 \cdot 8)$ & $0 \cdot 14$ \\
\hline HPD (b) & & $2 \cdot 1$ & $(1 \cdot 5-3 \cdot 0)$ & $<0.001$ & - & - & - & - & - & - \\
\hline MI (b) & & $0 \cdot 6$ & $(0 \cdot 4-0 \cdot 8)$ & $0 \cdot 02$ & $0 \cdot 01$ & $(0 \cdot 0-1 \cdot 7)$ & $0 \cdot 08$ & $0 \cdot 5$ & $(0 \cdot 1-2 \cdot 1)$ & $0 \cdot 38$ \\
\hline \multirow[t]{2}{*}{ Maturity } & Juveniles & Ref. & & & Ref. & & & Ref. & & \\
\hline & Adults & $3 \cdot 2$ & $(1 \cdot 3-8 \cdot 3)$ & $0 \cdot 04$ & $7 \cdot 9$ & $(0 \cdot 6-100)$ & $0 \cdot 11$ & $4 \cdot 6$ & $(0 \cdot 8-27)$ & $0 \cdot 03$ \\
\hline HPD (c) & & $2 \cdot 8$ & $(2 \cdot 0-4 \cdot 1)$ & $<0.001$ & - & - & - & - & - & - \\
\hline MI (c) & & $0 \cdot 4$ & $(0 \cdot 3-0 \cdot 7)$ & $0 \cdot 007$ & $0 \cdot 006$ & $(0 \cdot 0-0 \cdot 6)$ & $0 \cdot 03$ & $0 \cdot 6$ & $(0 \cdot 1-2 \cdot 1)$ & $0 \cdot 41$ \\
\hline \multirow[t]{2}{*}{ Maturity } & Juveniles & Ref. & & & Ref. & & & Ref. & & \\
\hline & Adults & $3 \cdot 8$ & $(1 \cdot 5-9 \cdot 3)$ & $0 \cdot 01$ & $6 \cdot 8$ & $(0 \cdot 5-84)$ & $0 \cdot 13$ & $4 \cdot 3$ & $(0 \cdot 7-27 \cdot 9)$ & $0 \cdot 126$ \\
\hline HPD (d) & & $2 \cdot 3$ & $(1 \cdot 6-3 \cdot 4)$ & $<0.001$ & - & - & - & - & - & - \\
\hline MI (d) & & $0 \cdot 5$ & $(0 \cdot 3-0 \cdot 8)$ & $0 \cdot 02$ & 0.02 & $(0 \cdot 0-0 \cdot 4)$ & 0.009 & $0 \cdot 7$ & $(0 \cdot 2-2 \cdot 6)$ & $0 \cdot 63$ \\
\hline
\end{tabular}

aOR, Adjusted odds ratio; CI, confidence interval.

* aOR, $95 \% \mathrm{CI}$ and $P$ values obtained using the final $L$. interrogans GLM.

$\dagger$ aOR, 95\% CI and $P$ values obtained using the final SEOV GLMM.

$\$ \mathrm{aOR}, 95 \% \mathrm{CI}$ and $P$ values obtained using the final HEV GLMM.

mature rats than sexually immature rats. The relationships between eight normalized socioeconomic covariates and HEV positivity were statistically significant (all $P$ values $\leqslant 0 \cdot 05$ ).

The multivariate analyses used sexual maturity, HPD, and income as independent covariates. In the final GLM, sexual maturity and normalized income were retained. The OR of being HEV-positive increased with decreasing normalized income (OR $0 \cdot 4,95 \%$ CI $0 \cdot 2-0 \cdot 6)$ and appeared to be greater in adults than juveniles, although results in the latter group were not statistically significant (OR 2.2, 95\% CI $0 \cdot 7-7$ ). In bivariate analyses, the relationship between these variables and HEV positivity aligned but with increased magnitude. Similar results were obtained by accounting for clustering trapping locations using a GLMM. In the selected GLMM, the estimated variance for the random effect of trapping locations was 1.631 , accounting for $7 \%$ of the total residual variance.

\section{T. gondii exposure}

The results from the SLR models suggested that only body mass was significantly higher in rats exposed to
T. gondii $(P \leqslant 0 \cdot 05)$. Thus, the multivariate analysis was not investigated.

\section{Test of the spatial extents of remotely sensed extracted data}

The new datasets extracted from the four quadrats were incorporated into the final models selected for L. interrogans infection status (see Supplementary online material). The $P$ values associated with the relationship between infection status and the covariates decreased with increasing quadrat size. Larger quadrats displayed a better goodness-of-fit model (Table 4). Spatial distributions of the covariates are shown in Figure 2; areas of higher and lower levels of $L$. interrogans infection were mainly located in densely populated and low-income areas.

\section{DISCUSSION}

This study evaluated the risks of four potentially zoonotic pathogens in Norway rats in Lyon. We demonstrated that rats in human habitats were infected or exposed and probably shed L. interrogans, SEOV, $\mathrm{HEV}$, and T. gondii. Based on logistic regression 


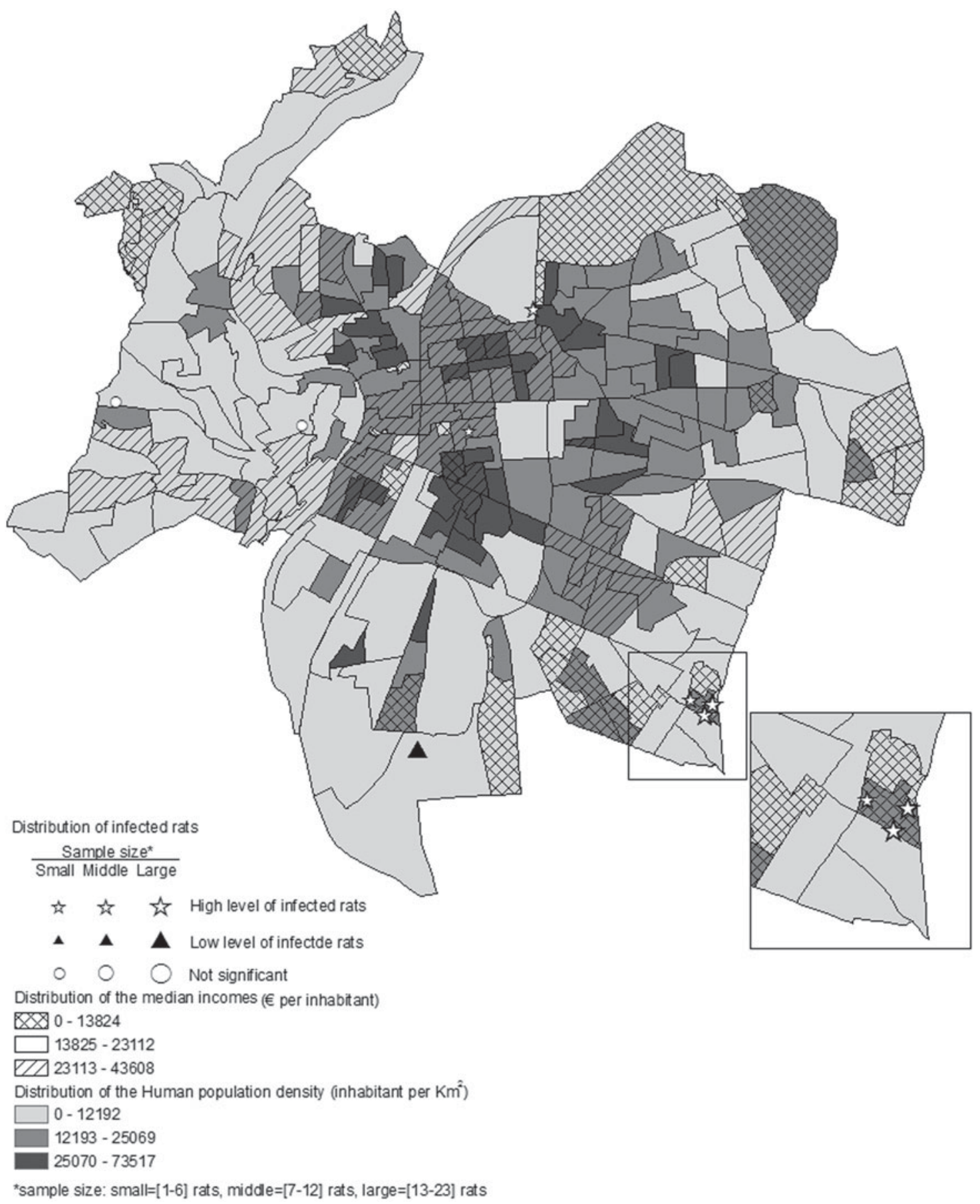

Fig. 2. The spatial distribution of human population density and median incomes in Lyon city centre overlaid with high and low prevalence of $L$. interrogans carriage in Norway rats.

models, our study suggests that populated and/or low-income areas of the Rhône department may encompass an increased number of infected rats. Below, the spatially explicit approach and detection methods are expanded upon to explain how the identified risk factors might be used in targeted surveillances.

\section{Spatial analysis methodology}

The risk analysis demonstrated an overlap of densely populated, low-income urbanized areas and Norway rats with $L$. interrogans, SEOV, and HEV infections. Socioeconomic data collected by the INSEE census are most commonly aggregated at the administrative 
boundary or census areal unit level. Spatial scale can affect the strength and significance of statistical associations, which is known as the modifiable areal unit problem (MAUP) [23]. To minimize the MAUP effect in statistical inference, the covariates can be analysed at hierarchical levels of areal units from the finest to the coarsest resolution to confirm consistent model results [24]. Five hierarchical levels of census units were used in this study. Only the smallest unit failed to confirm the relationship between socioeconomic data and $L$. interrogans positivity, suggesting that HPD and median incomes are robust covariates in predicting the $L$. interrogans infection status in rats, as the ORs were similar when the spatial scale was changed. The ability of HPD and income to predict $L$. interrogans infection in rats aligns with previous studies demonstrating the distribution of human leptospirosis in impoverished inner-city areas [25].

\section{Pathogen detection}

Generally, screening tests used in wildlife species were developed for humans or domestic species and do not have gold standards, as in the case of wild rats. Bacteria recovery from an individual kidney by bacteriological culture was used as the definitive diagnostic of $L$. interrogans and was considered to be appropriate for early detection because kidney colonisation occurs in the first week of Leptospira infection [26]. However, this culturing method is insensitive, and the bacterial isolation frequency is typically low. Thus, qPCR was also performed because it is reportedly the most sensitive human analysis [27]. False negatives can still be obtained when PCR is used for undescribed pathogenic strains [28] or the bacteria are aggregated in an unsampled part of the kidney. Therefore, a combination of culture and qPCR was relevant; one positive culture and negative PCR were observed in this study. This combination is expected to provide a reliable reflection of Leptospira infection in rats. Nevertheless, we found that negative tests were more likely to occur in dead rats for which PCR inhibitors might be responsible for false-negative results [29]. This result suggests that the prevalence, although consistent with a previous survey in France, might be underestimated [4].

The previously reported sequences of SEOV and HEV [18, 19] demonstrate their presence in individuals. Although several lung and liver lobes were sampled, detection may fail when the viral load is not homogeneously distributed throughout the organs, and areas clear of infection are collected. The combination of HEV-PCR detection on liver and faeces optimized HEV RNA detection; indeed, two individuals were liver-negative and faecespositive, suggesting that this study may provide an appropriate reflection of rat infection. In addition, the observed prevalence was consistent with previous surveys $[30,31]$. Conversely, the prevalence of SEOV might be underestimated.

In domestic animals, the MAT titres of 1:20-1:25 have been validated to detect $\operatorname{IgG}$ antibodies to $T$. gondii in sera [32]. The choice of cut-off is usually raised when interpreting serological tests developed for domestic species or humans. In other wildlife species, 1:6 is a biologically relevant threshold because many individuals with titres of 1:6 carry Toxoplasma cysts [33]. However, given the low antibody levels of some individuals, all individuals were bioassayed when possible. The absence of Toxoplasma detection in mice could be caused by a previous infection that resulted in persistent antibodies, the absence of the parasite in the heart, or parasite death during sample transport. Nonetheless, the $8 \%$ of rats exposed to $T$. gondii in the Lyon region and the variation between sites was in agreement with a recent survey in France [34].

\section{Towards a RBZ-targeted surveillance}

Considering the propensity of rats to carry zoonotic pathogens, a public health surveillance programme should be developed. However, using aggregated data may misestimate the prevalence, as aggregate data have a heterogeneous spatial distribution. Thus, risk-based surveillance combining the risk of zoonotic pathogen shedding in rats and the risk of rat-human proximity should be developed to assess and mitigate the spread of RBZs. As the transmission of the four pathogens in rats relies on direct (i.e. behavioural) and indirect (i.e. environmental) contacts, both features should be considered to target surveillance and interpret results.

The zoonosis carriage risk in rats appears to be influenced by the colony's age structure. In our study, sexual maturity (particularly increased age) was identified as a risk factor for L. interrogans, SEOV, and HEV, as previously described $[35,36]$. The potential influence of age on infection status may result from the presence of maternal antibodies, which can persist for several months in rat neonates [37]. Rat infections are also more likely to be acquired with exploratory 
behaviour development, which increases with sexual maturity. No male bias was revealed in this study although the likelihood of males acquiring SEOV would be greater due to them having more aggressive encounters and as wounds would be the primary route of SEOV transmission between adult males [38]. Nevertheless, a longitudinal study on rodents revealed that the male bias observed in the prevalence of antibody to hantaviruses varied among trapping sites and was more likely due to habitat, social structure or behavioural variations [39]. The absence of male bias in the present study may be related to its short-term design or to site criteria. Thus, stratified sampling and the colony's age structure should be considered to provide a relevant estimate of $\mathrm{RBZ}$ carriage.

We observed a robust relationship between rat infection and socioeconomic covariates which more likely results from a confounding effect of the socioeconomic factors with genuine causal factors. The micro-environment features are suspected to be a causal factor involved in pathogen transmission. As Himsworth et al. suggested regarding the relationship between micro-environmental features and Leptospira prevalence (i.e. environmental factors likely related to variations in Leptospira prevalence) [40], our study additionally suggests to focus on environments associated with dense populations and areas with lower incomes. In this way, the results obtained partially answer a previous report on RBZs highlighting the necessity for public health officials to define environmental features that promote pathogen transmission in rats [41]. By suggesting the infected rat distribution patterns, the socio-economic factors could aid targeting and enhancing surveillance. In addition, the effect of socio-economic factors could result from the related effect of rat population size which may increase in lower income areas because of lower hygiene and lower pest control measures. In our study, the effect of the capture success on the number of infected rats was not revealed and could be explained by its low ability to estimate the population size or specific transmission schemes not population-size-related. Indeed, the rat capture success does not merely depend on population size but also on resources, shelter, age structure of the population or pre-poisoning. Furthermore, the usual method of rodent trapping using grids or transects may not be adapted to urban environments since there are a number of physical barriers as well as adverse effects of human activities. The understanding of the potential ways of pathogen transmissions (vertical vs. horizontal, direct contact $v s$. environment) and their relative importance is limited. The population density effect on pathogen prevalence might not be the rule in rat colonies as previously suggested for $L$. interrogans infections [40] although it may depends on the pathogens or colonies. As there is no standard to assess the urban rat population size, a critical lack of information limits our study to exploratory work dedicated to suggesting new approaches for the study of RBZ risks.

\section{CONCLUSION}

This study enhances knowledge of the spatial distribution of zoonotic pathogens in Norway rats from the Rhône department. The results suggest that the risk of RBZs is greater in densely populated and lower income areas of Rhône. Given the unprecedented rate of global urbanization and poverty, this study suggests the development of appropriate risk-based surveillance of zoonotic pathogens in Norway rats by targeting reliable indicators of areas with high exposure risks. Although further investigations are required to confirm and refine these findings and to assess the exposure risk in humans, this type of surveillance is required to assess and mitigate the spread of RBZs and to control their potential transmission to humans.

As part of a comprehensive risk assessment, this study should improve prevention measures in the context of a typical 'One Health' approach.

\section{SUPPLEMENTARY MATERIAL}

For supplementary material accompanying this paper visit http://dx.doi.org/10.1017/S0950268814001137.

\section{ACKNOWLEDGEMENTS}

We thank the city of Lyon and its 'Service d'hygiène' (Dr. Gilbert Gault), the waste plant UIOM de Lyon (M Joël Baud), the Parc de la Tête d'Or managers (Dr. David Gomis and Dr. Guillaume Douay), and the Agence d'urbanisation for assisting with rat and census data collection. We thank Philippe Berny and Joffrey Goullois for assisting with rat collections, Emmanuelle Gilot-Fromont for assisting with diagnosis and analysis, Zorée Djelouadji and Lorraine McElhinney (AHVLA) for assisting with screening for Leptospira and Seoul virus, respectively, and the Institut National Geographique et Forestière (IGN) 
for providing census data. The hantavirus identification was funded by the Defra Surveillance Project SV3037.

This project was supported by the European Commission under the Food, Agriculture and Fisheries and the Biotechnology theme of the Seventh Framework Programme for Research and Technological Development (grant no. 222633), WildTech project WP6 Epidemiology and Risk assessment.

\section{DECLARATION OF INTEREST}

None.

\section{REFERENCES}

1. Jones KE, et al. Global trends in emerging infectious diseases. Nature 2008; 451: 990-993.

2. Meerburg BG, Singleton GR, Kijlstra A. Rodent-borne diseases and their risks for public health. Critical Reviews in Microbiology 2009; 35: 221-270.

3. Vijayachari P, Sugunan AP, Shriram AN. Leptospirosis: an emerging global public health problem. Journal of Biosciences 2008; 33: 557-569.

4. Aviat F, et al. Leptospira exposure in the human environment in France: a survey in feral rodents and in fresh water. Comparative Immunology, Microbiology, and Infectious Diseases 2009; 32: 463-476.

5. Socolovschi C, et al. Strikes, flooding, rats, and leptospirosis in Marseille, France. International Journal of Infectious Diseases 2011; 15: e710-e715.

6. Plyusnin A, Vapalahti O, Vaheri A. Hantaviruses: genome structure, expression and evolution. The Journal of General Virology 1996; 77: 2677-2687.

7. Jameson LJ, et al. The continued emergence of hantaviruses: isolation of a Seoul virus implicated in human disease, United Kingdom, October 2012. Eurosurveillance 2013; 18: 4-7.

8. Macé G, et al. Severe Seoul hantavirus infection in a pregnant woman, France, October 2012. Eurosurveillance 2013; 18: 20464.

9. Krüger DH, Schönrich G, Klempa B. Human pathogenic Hantaviruses and prevention of infection. Human Vaccines 2011; 7: 685-693.

10. Olsson GE, et al. Demographic factors associated with hantavirus infection in bank voles (Clethrionomys glareolus). Emerging Infectious Diseases 2002; 8: 924-929.

11. Johne $\mathbf{R}$, et al. Novel hepatitis $E$ virus genotype in Norway rats, Germany. Emerging Infectious Diseases 2010; 16: 1452-1455.

12. Wolf S, et al. The simultaneous occurrence of human norovirus and Hepatitis $\mathrm{E}$ virus in Norway rats (Rattus norvegicus). Archives of Virology 2013; 158: 1575-1578.
13. Lack JB, Volk K, Van Den Bussche RA. Hepatitis E virus genotype 3 in wild rats, United States. Emerging Infectious Diseases 2012; 18: 1268-1273.

14. Jones J, Lopez A, Wilson M. Congenital toxoplasmosis. American Family Physician 2003; 67: 2131-2138.

15. Sarkar U, et al. Population-based case-control investigation of risk factors for leptospirosis during an urban epidemic. American Journal of Tropical Medicine and Hygiene 2002; 66: 605-610.

16. Thurdfield M. Veterinary Epidemiology, 3rd edn. Oxford: Blackwell Science Ltd, 2007, pp. 232-242.

17. Zuerner RL. Laboratory maintenance of pathogenic Leptospira. Current Protocols in Microbiology 2005; chapter 12: unit 12E.1.

18. Dupinay T, et al. Detection and genetic characterization of Seoul virus from commensal brown rats in France. Virology Journal 2014; 11:32.

19. Widèn F, et al. Detection of potentially zoonotic hepatitis $\mathrm{E}$ virus in rats captured in an urban area in Lyon France during 2011 and 2012. Virology Journal (in press).

20. Dubey JP, Desmonts G. Serological responses to equids fed Toxoplasma-gondii oocysts. Equine Veterinary Journal 1987; 19: 337-339.

21. Villena I, et al. Evaluation of a strategy for Toxoplasma gondii oocyst detection in water. Applied and Environmental Microbiology 2004; 70: 4035-4039.

22. Sokal RR, Rohlf FS. Biometry. New York: W. H. Freeman, 1981.

23. Diez-Roux AV. Multilevel analysis in public health research. Annual Review of Public Health 2000; 21: 191-192.

24. Unwin DJ. GIS spatial analysis and spatial statistics. Progress in Human Geography 1996; 20: 540.

25. Oliveira DS, et al. The socio-demographic, environmental and reservoir factors associated with leptospirosis in an urban area of north-eastern Brazil. Annals of Tropical Medicine and Parasitology 2009; 103: 149-157.

26. Athanazio DA, et al. Rattus norvegicus as a model for persistent renal colonisation by pathogenic Leptospira interrogans. Acta Tropica 2008; 105: 176-180.

27. Bharti AR, et al. Leptospirosis: a zoonotic disease of global importance. Lancet Infectious Diseases 2003; 3: 757-771.

28. Bourhy $\mathbf{P}$, et al. Comparison of real-time PCR assays for detection of pathogenic Leptospira spp. in blood and identification of variations in target sequences. Journal of Clinical Microbiology 2011; 49: 2154-2160.

29. Alaeddini R. Forensic implications of PCR inhibitiona review. Forensic Science International: Genetics 2012; 6: $297-305$.

30. Zhang YZ, et al. Hantavirus infections in humans and animals, China. Emerging Infectious Diseases 2010; 16: 1195-1203.

31. Kanai Y, et al. Hepatitis E virus in Norway rats (Rattus norvegicus) captured around a pig farm. BMC Research Notes 2012; 5: 4.

32. Dubey JP. Validation of the specificity of the modified agglutination test for toxoplasmosis in pigs. Veterinary Parasitology 1997; 71: 307-310. 
33. Richomme C, et al. Genetic characterization of Toxoplasma gondii from wild boar (Sus scrofa) in France. Veterinary Parasitology 2009; 164: 296-300.

34. Gotteland C, et al. Species or local environment, what determines the infection of rodents by Toxoplasma gondii? Parasitology 2013; 18: 1-10.

35. Easterbrook JD, et al. A survey of zoonotic pathogens carried by Norway rats in Baltimore, Maryland, USA. Epidemiology and Infection 2007; 135: 1192-1199.

36. Hannah MF, Bajic VB, Klein SL. Sex differences in the recognition of and innate antiviral responses to Seoul virus in Norway rats. Brain, Behavior, and Immunity 2008; 22: 503-516.

37. Dohmae K, Nishimune Y. Protection against hantavirus infection by dam's immunity transferred vertically to neonates. Archives of Virology 1995; 140: 165-172.
38. Hinson ER, et al. Wounding: the primary mode of Seoul virus transmission among male Norway rats. American Journal of Tropical Medicine and Hygiene 2004; 70: 310-317.

39. Calisher CH, Wagoner K. Demographic factors associated with prevalence of antibody to Sin Nombre virus in deer mice in the Western United States. Journal of Wildlife Diseases 2007; 43:1-11.

40. Himsworth CG, et al. Ecology of Leptospira interrogans in Norway rats (Rattus norvegicus) in an inner-city neighborhood of Vancouver, Canada. PLoS Neglected Tropical Diseases 2013; 7: e2270.

41. Himsworth CG, et al. Rats, cities, people, and pathogens: a systematic review and narrative synthesis of literature regarding the ecology of rat-associated zoonoses in urban centers. Vector Borne Zoonotic Diseases 2013; 13: 349-359. 